\title{
Effect of CUL4A on the metastatic potential of lung adenocarcinoma to the bone
}

\author{
PEI-PEI CAO ${ }^{1 *}$, WEN-JUAN CHEN ${ }^{2 *}$, HAI-LIN PANG ${ }^{1}$, WEI-WEI SHEN ${ }^{1}$, PENG XUE $^{3}$, \\ LIAN DUAN $^{1}$, YU-XUAN XU ${ }^{1}$, LI-LI LIU ${ }^{1}$ and HE-LONG ZHANG ${ }^{1}$ \\ ${ }^{1}$ Department of Oncology, Tangdu Hospital, The Air Force Medical University, Xi'an, Shaanxi 710038; \\ ${ }^{2}$ Department of Oncology, Tumor Hospital of Shaanxi Province, Xi'an, Shaanxi 710061; \\ ${ }^{3}$ Department of Orthopedics, Honghui Hospital, Xi'an Jiaotong University, Xi'an, Shaanxi 710054, P.R. China
}

Received June 28, 2019; Accepted November 27, 2019

DOI: $10.3892 /$ or.2019.7448

\begin{abstract}
Cullin $4 A(C U L 4 A)$ is a member of the cullin family of proteins and has been demonstrated to be abnormally expressed in various types of malignancies. However, the function of $C U L 4 A$ in metastasis of lung adenocarcinoma to the bone has rarely been reported. The aim of present of the study was to explore the biological functions and potential underlying molecular mechanisms of CUL4A in lung adenocarcinoma, highlighting a novel therapeutic target for the diagnosis and treatment of patients with lung adenocarcinoma. A549-CUL4A, H1299-CUL4A and H460-shCUL4A cells were created using lentiviral infection. The efficiency of knockdown or overexpression was assessed using reverse transcription-quantitative PCR and western blotting. The effects of $C U L 4 A$ on proliferation, migration and invasion of lung adenocarcinoma cells in vitro and metastasis to the bone in vivo were determined using an MTT assay, colony formation assay, wound-healing assay, Transwell assay and a mouse model of bone metastasis. The relationship between CUL4A and the EMT-activator zinc finger E-box binding homeobox 1 (ZEB1) were detected by western blotting. The results showed that overexpression of $C U L 4 A$ in lung adenocarcinoma cells increased proliferation, migration and invasion, and increased metastasis of A549 to the bones in vivo. Silencing of CUL4A expression in lung adenocarcinoma cells reduced proliferation, migration and invasion in vitro. Mechanistically, CUL $4 A$ transcriptionally upregulated expression of $Z E B 1$ which resulted in epithelial-mesenchymal transition, which in turn
\end{abstract}

Correspondence to: Professor He-Long Zhang, Department of Oncology, Tangdu Hospital, The Air Force Medical University, 569 Xinsi Road, Xi'an, Shaanxi 710038, P.R. China

E-mail: cnxazhl@163.com

${ }^{*}$ Contributed equally

Key words: CUL4A, cullin 4A, lung adenocarcinoma, ZEB1, epithelial-mesenchymal transition, bone metastasis promoted metastasis of lung adenocarcinoma to the bones. Taken together, these results suggest that $C U L 4 A$ may serve an important regulatory role in the development of metastasis of lung adenocarcinoma to the bone.

\section{Introduction}

Non-small cell lung cancer (NSCLC) is the most common type of lung cancer and accounts for $\sim 85 \%$ of all lung cancer cases, and is one of the leading causes of cancer-associated mortality worldwide (1-3). Adenocarcinoma is the fastest growing subtype of NSCLC, and the 5-year survival rate is <20\% (4), and 30-40\% of patients with advanced stage lung cancer develop bone metastases, resulting in skeletal-related events which, in-turn cause hypercalcemia, pathological fractures, spinal compression and bone pain, leading to poor prognoses (5). Exploring the underlying molecular mechanisms of lung cancer metastasis to the bone has gained increasing interest, particularly for the exploration of novel therapeutic targets.

Cullin $4 A(C U L 4 A)$ is an $87-\mathrm{kDa}$ protein and is a member of the cullin family of proteins. $C U L 4 A$ forms part of the multifunctional ubiquitin ligase E3 complex (6). The ubiquitin-proteasome pathway serves an important role in the degradation of proteins, including several well-defined tumor-suppressor genes, such as $p 21, p 27$ and $p 53$ (7). Additionally, it has been reported that $C U L 4 A$ is abnormally expressed in various types of malignancies (7). Therefore, $C U L 4 A$ may act as an oncogene to promote tumor progression; however, the association between $C U L 4 A$ and metastasis of lung adenocarcinoma to the bone has not been reported.

Epithelial-mesenchymal transition (EMT) is the initial event in the tumor metastatic process, which promotes the dissemination of tumor cells from the primary lesion to colonization at distant sites (8). Zinc finger E-box binding homeobox $l(Z E B 1)$ is a transcriptional activator of EMT, and it represses expression of epithelial genes by binding to the promoter regions of E-boxes, inducing EMT and thus promoting cancer metastasis (9). Furthermore, ZEBI has been reported to promote metastasis of lung cancer to the bone in vivo (10). Therefore, clarifying the association between $C U L 4 A$ and ZEB1 may improve our understanding of metastasis of lung cancer to the bone. 
The present study revealed that $C U L 4 A$ overexpression promoted proliferation, migration and invasion of lung adenocarcinoma cells in vitro and metastasis of lung cancer to the bones in vivo. Knockdown of $C U L 4 A$ had the opposite effects on the biological behaviors of lung adenocarcinoma cells in vitro. Mechanistically, CUL4A induced EMT and promoted metastasis of lung adenocarcinoma to the bone by regulating the transcriptional expression of $Z E B 1$. These results provide novel insight into the mechanistic role of $C U L 4 A$ in metastasis of lung adenocarcinoma to the bone, suggesting that $C U L 4 A$ may serve as a potential therapeutic target for patients with advanced lung adenocarcinoma.

\section{Materials and methods}

Cell lines and cell culture. The human lung adenocarcinoma cell lines A549, H1299 and H460 were purchased from the American Type Culture Collection (ATCC) and have been preserved in our laboratory in a liquid nitrogen storage tank. Cells were grown in culture flasks with RPMI-1640 medium (Gibco; Thermo Fisher Scientific, Inc.) containing 10\% FBS (Biochrom, Ltd.) with $5 \% \mathrm{CO}_{2}$ at $37^{\circ} \mathrm{C}$ in an incubator.

Reverse transcription-quantitative $(R T-q) P C R$. Total RNA was extracted from cells using TRIzol ${ }^{\circledR}$ reagent (Invitrogen; Thermo Fisher Scientific, Inc.) and reverse-transcribed into cDNA using a QuantiNova ${ }^{\mathrm{TM}}$ Reverse Transcription kit (Qiagen $\mathrm{GmbH}$ ) according to the manufacturer's protocol. The quantification of gene transcripts was determined using qPCR using a QuantiNova ${ }^{\mathrm{TM}}$ SYBR Green PCR kit (Qiagen $\mathrm{GmbH}$ ) on a Mx3005P qPCR system (Agilent Technologies, Inc.). The thermocycling conditions were: Pre-denaturation at $95^{\circ} \mathrm{C}$ for $2 \mathrm{~min}$; followed by 40 cycles of denaturation at $95^{\circ} \mathrm{C}$ for $10 \mathrm{sec}$ and annealing at $60^{\circ} \mathrm{C}$ for $30 \mathrm{sec}$. Gene expression was quantified using the $2^{-\Delta \Delta \mathrm{Cq}}$ method (11). The sequences of the PCR primers were as follows: $C U L 4 A$ forward, GGC TCCAAGAAGCTGGTCAT and reverse, CTGATGGAG GTGCTGCTCTG; GAPDH forward, GAAGGTGAAGGT CGGAGTC and reverse, GAAGATGGTGATGGGATTTC. $G A P D H$ was used as the internal control.

Protein extraction and western blot analysis. Total protein was extracted from cells using RIPA lysis buffer (Beyotime Institute of Biotechnology Biotechnology), and the protein concentration was quantified using a bicinchoninic acid protein assay kit (Pierce; Thermo Fisher Scientific, Inc.). Subsequently, $20 \mu \mathrm{g}$ of protein was loaded on a $10 \%$ SDS gel and resolved using SDS-PAGE. Resolved proteins were transferred to PVDF membranes, and the membranes were blocked in 5\% fat-free milk for $1 \mathrm{~h}$ at room temperature and incubated overnight at $4^{\circ} \mathrm{C}$ with specific primary antibodies against CUL4A (dilution 1:500; cat. no. 14851-1-AP; ProteinTech Group, Inc.), $\beta$-actin (dilution 1:10,000; cat. no. 051M4892; Sigma-Aldrich; Merck KGaA), E-cadherin (dilution 1:1,000; cat. no. MABT26; Merck KGaA), Vimentin (dilution 1:1,000; cat. no. MABT26; Merck KGaA), ZEB1 (dilution 1:500; cat. no. 21544-1-AP; ProteinTech Group, Inc.). After incubation with the primary antibodies, the membranes were incubated with horseradish peroxidase-conjugated goat anti-mouse/rabbit secondary antibody (dilution 1:2,500; Beijing Zhongshan
Jinqiao Biotechnology Co., Ltd.) for $1 \mathrm{~h}$ at room temperature, and signals were visualized using chemiluminescence reagent (Pierce; Thermo Fisher Scientific, Inc.) and analyzed using AlphaImager 2200 software version 3.2.1.2 (Alpha Innotech Corporation). $\beta$-actin was used as the loading control.

Stable transfection. Short hairpin (sh)RNAs (CUL4A-shRNA28399-1, 5'-GCAGAACTGATCGCAAAG CAT-3'; CUL4A-shRNA28400-1, 5'-CCAGAATATCTTAAC CATGTA-3'; CUL4A-shRNA28402-1, 5'-GCAGGTGTATAA AGATTCATT-3') targeting CUL4A (CUL4A-GV248-RNAi NM_001008895, target sequence: GCAGAACTGATCGCA AAGCAT), control shRNA (NC-GV248, target sequence: TTCTCCGAACGTGTCACGT), recombinant CUL $4 A$ lentivirus (homo; NM_001008895), CUL4A-NC lentivirus (Ubi-MCS-3FLAG-SV40-puromycin) and Luciferin-LV (Ubi-MCS-Luc-IRES-Puromycin) were synthesized by Shanghai GeneChem Co., Ltd. (Shanghai, China), with virus titers of $1 \times 10^{9}, 1 \times 10^{9}, 1 \times 10^{9}, 1 \times 10^{9}$ and $5 \times 10^{8} \mathrm{TU} / \mathrm{ml}$. The CUL4A overexpression lentiviral vector (CUL4A-LV) was respectively infected into A549 and $\mathrm{H} 1299$ cells at a multiplicity of infection (MOI) of 80 and 40 with complete medium containing ENi.S and Polybrene (Shanghai GeneChem Co., Ltd.). Similarly, H460 cells were infected with the CUL4A-shRNA lentivirus at a MOI of 100 to knock down $C U L 4 A$ expression, and NC-shRNA was used as the negative control. Transfections were performed according to the manufacturer's instructions. The infected cells were selected for 2 weeks using a medium with a concentration of $2.0 \mu \mathrm{g} / \mathrm{ml}$ puromycin to obtain stably transfected cells. Then the puromycin level in the culture medium was maintained at $1 \mu \mathrm{g} / \mathrm{ml}$. Luciferin-LV virus was used to infect both A549-CUL4A and A549-NC cells for viewing the distribution of tumor cells in vivo. The efficiency of knockdown or overexpression was assessed using RT-qPCR and western blotting.

MTT assay. Cells in the logarithmic growth phase were collected and seeded into 96-well plates at a density of $2 \times 10^{3}$ cells/well. A total of eight 96 -well plates were cultured as described above. On the following days, a 96-well plate was taken out at a fixed daily time every $24 \mathrm{~h}$, MTT solution $(5 \mathrm{mg} / \mathrm{ml})$ was added $(20 \mu \mathrm{l} /$ well $)$, and the plate was incubated at $37^{\circ} \mathrm{C}$ for a further $4 \mathrm{~h}$. The medium was carefully discarded and $150 \mu \mathrm{l}$ of DMSO was added. The 96-well plate was agitated for $10 \mathrm{~min}$ to dissolve the formamidine completely, and the absorbance value was measured at a wavelength of $490 \mathrm{~nm}$ on a microplate reader (Multiskan MK3; Thermo Fisher Scientific, Inc.). This assay was performed in triplicate.

Colony formation assay. The cells in the logarithmic growth phase were harvested and plated in a 6-well plate at a density of 200 cells/well, and the plate was incubated as described above for two weeks. Subsequently, the cells were stained with $0.25 \%$ crystal violet for $20 \mathrm{~min}$ at room temperature. Subsequently, cell colonies (>50 cells) were counted manually. This assay was performed in triplicate.

Wound-healing assay. The cells in the logarithmic growth phase were plated in a 6 -well plate at a density of $5 \times 10^{5}$ cells/well and cultured in RPMI-1640 medium supplemented with $10 \%$ 
FBS, for $24 \mathrm{~h}$ until the cells reached $\sim 90 \%$ confluence. The cell monolayer was scratched using a $200-\mu 1$ pipette tip, and the cell debris was washed away with PBS. Then, the cells were cultured in serum-free RPMI-1640 medium for $24 \mathrm{~h}$. The wounds were imaged at $\mathrm{x} 4$ magnification using an inverted light microscope at 0 and $24 \mathrm{~h}$ after the scratch was made. The distance of the migration relative to the initial distance was calculated, and the migration distance was analyzed using ImageJ (version 1.8.0; ImageJ, Inc.). This assay was performed in triplicate.

Cell invasion assay. Transwell inserts $(8.0-\mu \mathrm{m}$ pore size) were coated with $70 \mu \mathrm{l}$ Matrigel (1:8 dilution; both from Corning Inc.). Cells in the logarithmic growth phase were harvested and resuspended to a density of $1 \times 10^{5}$ cells $/ \mathrm{ml}$ in serum-free RPMI-1640 medium. The single-cell suspension was plated into the upper chamber $(200 \mu \mathrm{l} /$ well $)$. A total of $500 \mu \mathrm{l}$ RPMI-1640 medium supplemented with 10\% FBS was added to the bottom chamber. The chambers were incubated as described above for $24 \mathrm{~h}$. Subsequently, the non-invading cells in the upper chamber were gently wiped off using cotton swabs, whereas cells that had invaded through the Matrigel were fixed in $95 \%$ ethyl alcohol for $5 \mathrm{~min}$ at room temperature and stained with $0.5 \%$ crystal violet for $20 \mathrm{~min}$ at room temperature. Subsequently, 10 randomly selected fields were imaged using a light microscope at x200 magnification, and the number of invaded cells were counted. This assay was performed in triplicate.

In vivo metastasis. All experiments involving animals were performed in accordance with the protocol approved by the Laboratory Animal Care of the Air Force Military Medical University (Xi'an, China). In the present study, 10 4-week-old female NOD-SCID mice weighing 15-17 g were obtained from Hunan SJA Laboratory Animal Co., Ltd. (http://zzx0251.bioon.com.cn/). Mice were randomly divided into two groups, each group consisting of 5 mice, and fed in a special pathogen-free grade animal facility at the Air Force Military Medical University. The mice were housed with a 12-h light/12-h dark cycle environment at $22^{\circ} \mathrm{C}$; ventilation rate, $15 / \mathrm{h}$; the food was sterilized with Cobalt-60 irradiation and water was autoclaved; and the mice had ad libitum access to food. A549-CUL4A and A549-NC cells in logarithmic growth phase were harvested with PBS to a single cell suspension with a density of $1.5 \times 10^{7}$ cells $/ \mathrm{ml}$. Single cell suspensions ( $3 \times 10^{6}$ cells $/ 200 \mu \mathrm{l}$ ) were injected into the mice via the tail vein. The health and progression of the tumor mass in the mice was examined weekly from the fifth week after injection onwards. When the experimental mice began to develop symptoms such as lameness, joint stiffness, decreased exercise capacity, paraplegia, or the experiment reached 42 days, the experiment was immediately terminated. D-Luciferin solution $(150 \mu \mathrm{l})$ (20 $\mathrm{mg} / \mathrm{ml}$ ) was intraperitoneally injected into the mice. After $10 \mathrm{~min}$, the mice were sacrificed humanely in a transparent euthanasia device (ventilated 3\% isoflurane for induction of anaesthesia and subsequent ventilated 1.5\% isoflurane for maintenance of anaesthesia) and placed in a prone position on the in vivo Imaging system (Carestream Health, Inc.) to capture X-ray images and biofluorescence imaging of the mice for examination of metastasis to the bone.
Statistical analysis. A Student's t-test or one-way ANOVA was used to analyze statistical differences of the effect of CUL $4 A$ on cell proliferation, colony formation, migration and invasion and data are presented as the mean \pm standard deviation of three replicates. A Wilcoxon rank sum test was used to analyze the bone metastasis data in vivo. Statistical tests were performed using SPSS (version 13.0.0; SPSS, Inc.). P<0.05 was considered to indicate a statistically significant difference.

\section{Results}

Expression of CUL4A in human lung adenocarcinoma cell lines. CUL4A expression was determined in A549, H1299 and $\mathrm{H} 460$ cells. The results showed that the expression level of CUL4A in the H460 cells was higher when compared with the A549 and H1299 cells (Fig. 1A and B). Furthermore, transfection efficiency of $C U L 4 A$ was analyzed using RT-qPCR and western blotting. CUL $4 A$ mRNA (Fig. 1C) and protein (Fig. 1D) expression levels in A549-CUL4A and H1299-CUL4A cells were both stably increased compared with the respective control cells, and the expression levels of $C U L 4 A$ in H460-shCUL4A cells were stably decreased compared with the parental H460-NC cells (Fig. 1E and F).

CUL4A increases the proliferative capacity of lung adenocarcinoma cells. The effect of CUL4A on the proliferative capacity of lung adenocarcinoma cells was determined using an MTT assay (Fig. 2A, C and E) and colony formation assays (Fig. 2B, D and F). Compared with the respective vector-only controls, both A549-CUL4A and H1299-CUL4A cells exhibited significantly increased cell proliferation and colony formation. Conversely, silencing of CUL4A expression in the H460 cells significantly reduced cell proliferation and colony formation compared with the control H460 cells.

CUL4A increases the migratory and invasive capacity of lung adenocarcinoma cells in vitro. The effect of CUL $4 A$ on cell migration was first assessed using a wound-healing assay (Fig. 3A). Both A549-CUL4A $(0.48 \pm 0.025)$ and H1299-CUL4A $(0.40 \pm 0.020)$ cells had significantly faster wound closure rates compared with the respective controls $(0.25 \pm 0.050$ and $0.33 \pm 0.025$, respectively), and conversely the wound closure rate of the H460-shCUL4A cells $(0.10 \pm 0.020)$ was slower compared with the respective control cells (0.18 \pm 0.029$)$. Additionally, A549-CUL4A

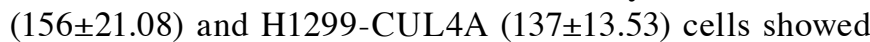
a greater degree of invasion in the Matrigel invasion assays compared with the respective control cells $(84 \pm 12.77$ and $68 \pm 16.65$, respectively; Fig. 3B and C). In contrast, silencing of CUL4A expression in $\mathrm{H} 460$ cells significantly reduced the invasive capacity of $\mathrm{H} 460$ cells $(82 \pm 11.00$ and $155 \pm 17.69$, respectively; Fig. 3D). These results indicate that $C U L 4 A$ promotes the migratory and invasive capacity of lung adenocarcinoma cells.

CUL4A overexpression facilitates bone metastasis in vivo. To explore the biological role of $C U L 4 A$ overexpression in the metastasis of lung adenocarcinoma to the bone, an experimental bone metastatic mouse model was constructed. A549-CUL4A cells were injected into NOD/SCID mice through the tail vein 
A

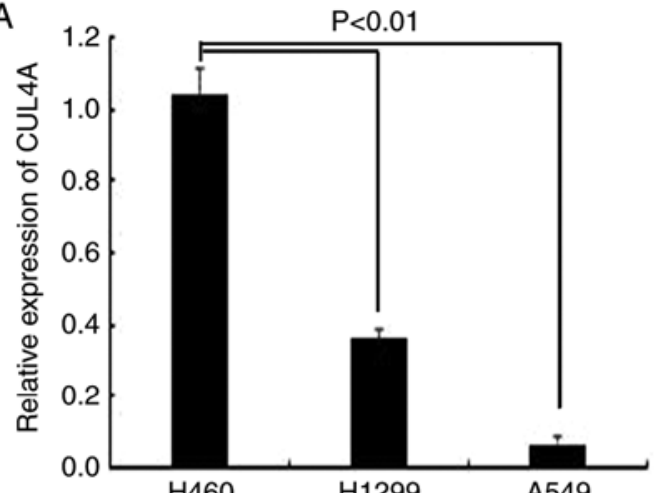

C
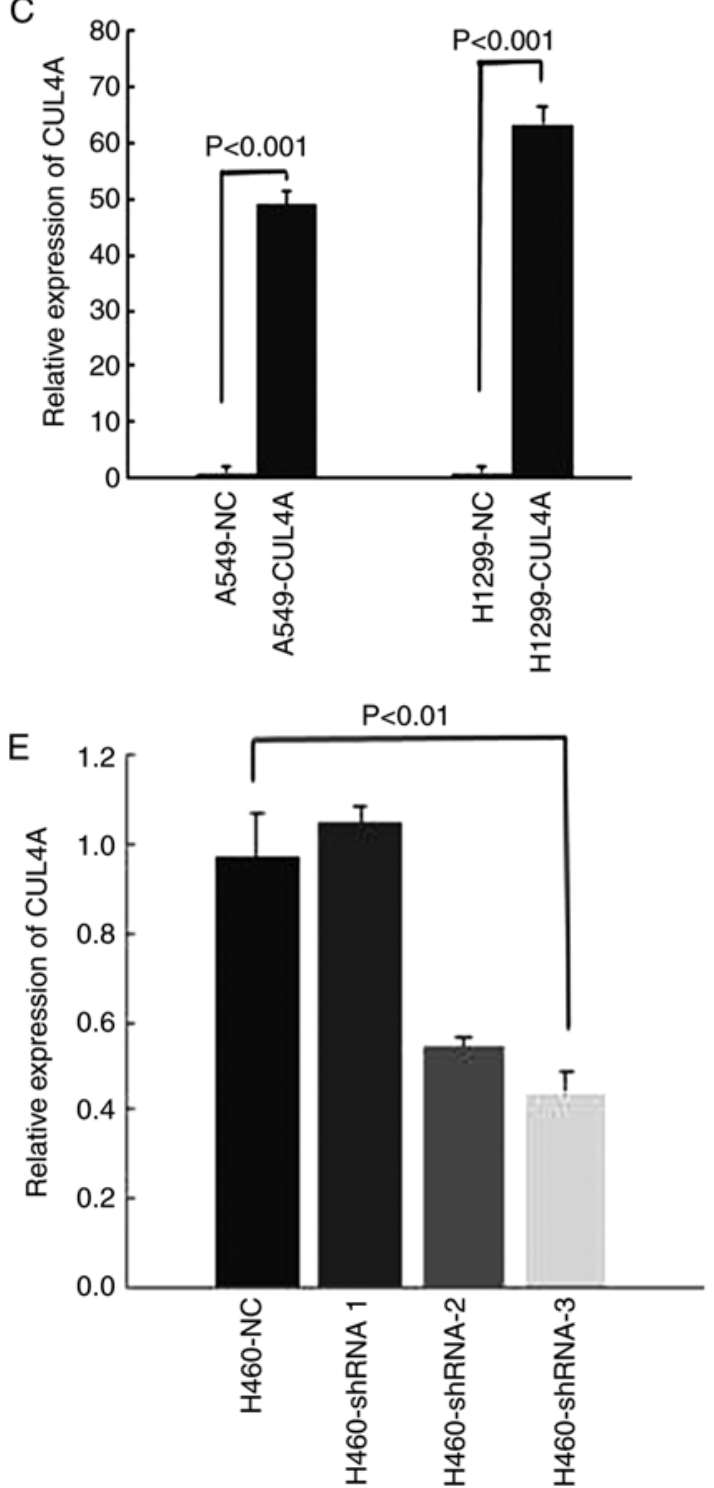

B

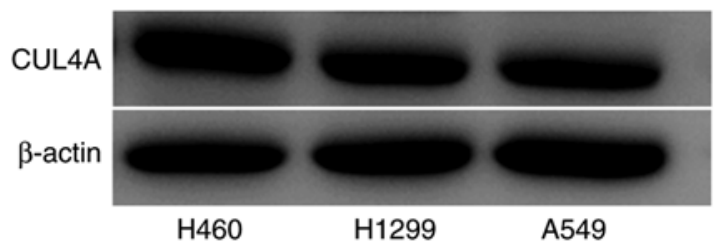

D

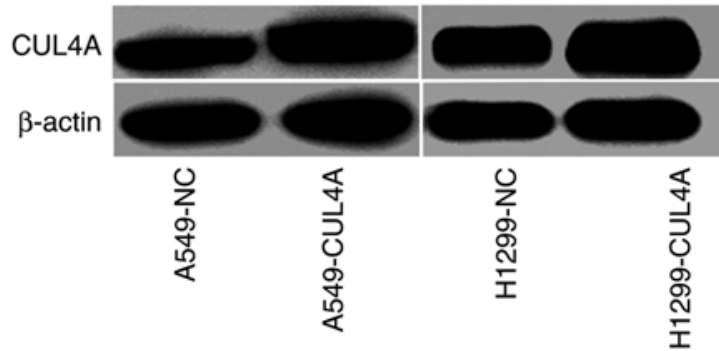

$\mathrm{F}$

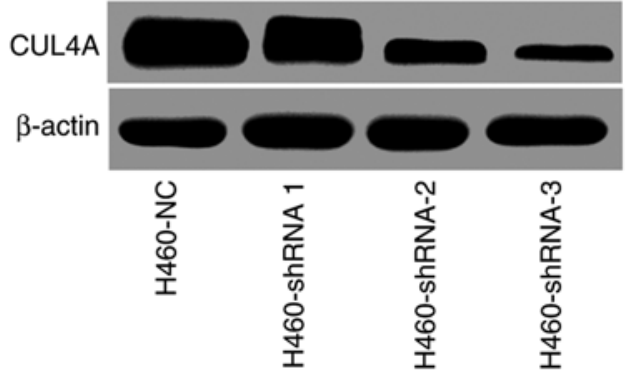

Figure 1. Expression levels of $C U L 4 A$ in lung adenocarcinoma cells. CUL4A expression was analyzed using (A) reverse transcription-quantitative PCR and (B) western blotting in A549, H1299 and H460 cells. P<0.01 vs. H460. (C) mRNA and (D) protein expression levels of CUL4A in cells transfected with a CUL4A overexpression vector. $\mathrm{P}<0.001$ vs. respective NC. (E) mRNA and (F) protein expression levels of CUL4A in cells transfected with the lentiviral knockdown vectors. $\mathrm{P}<0.001$ vs. respective NC. CUL4A, cullin $4 A ; \mathrm{NC}$, negative control; sh, short hairpin.

and the mice were assayed for the development of bone metastatic lesions. Compared with the control group, the injection of A549-CUL4A cells resulted in a significant increase in bone metastatic lesions (Fig. 4; Table I). Taken together, these results suggested that $C U L 4 A$ overexpression had the potential to promote the metastatic ability of lung adenocarcinoma bone metastasis in vivo.

CUL4A promotes metastasis of lung adenocarcinoma to the bone via ZEB1-mediated induction of EMT. In order to gain 
A

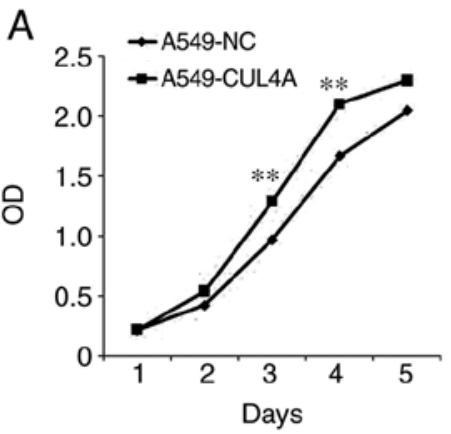

B

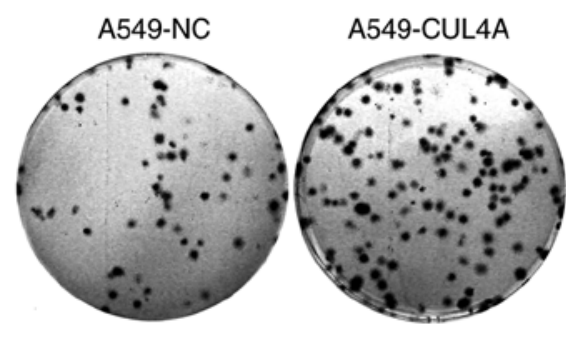

D

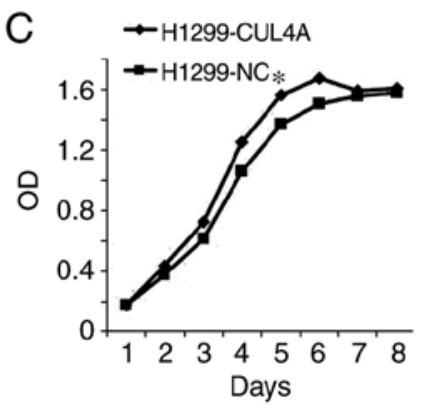

E

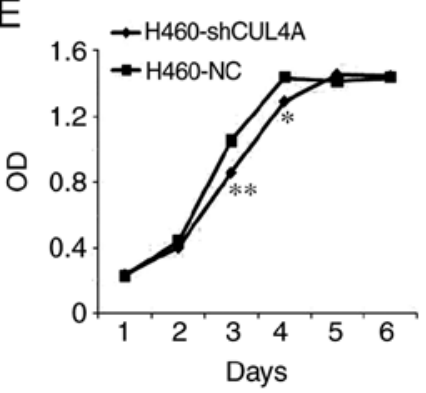

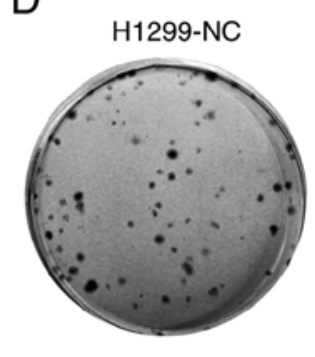

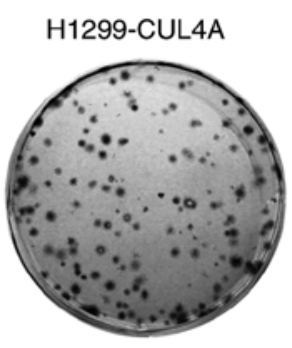

$\mathrm{F}$

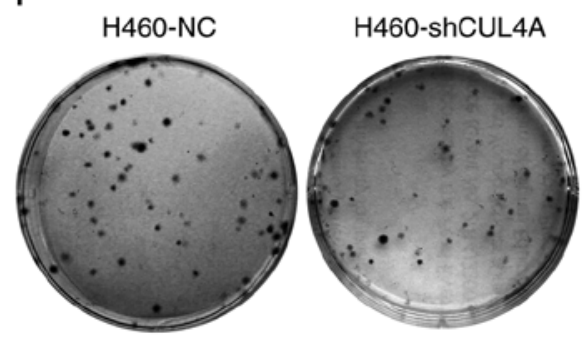

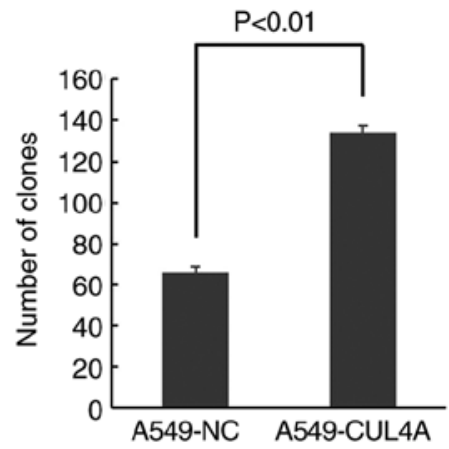
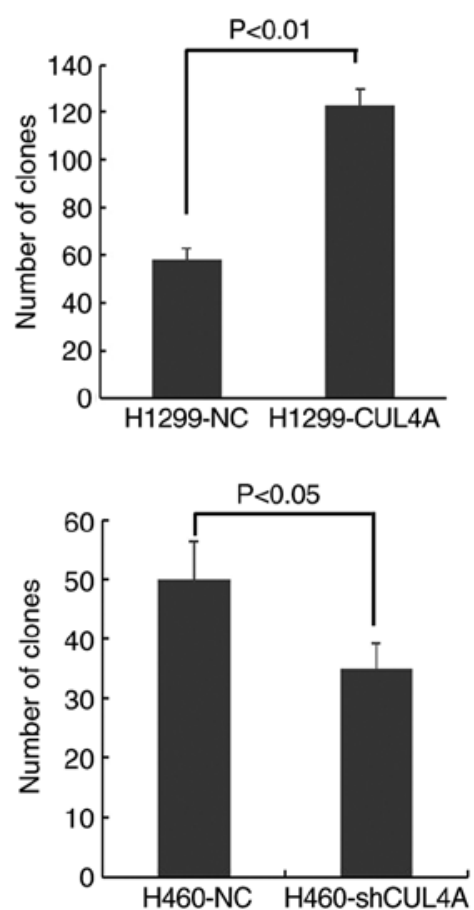

Figure 2. Effect of CUL4A on the proliferation of lung adenocarcinoma cells. (A) Proliferation of A549-NC and A549-CUL4A cells determined using an MTT assay. (B) Colony formation assays of A549-NC and A549-CUL4A cells. (C) Proliferation of H1299-NC and H1299-CUL4A cells determined using an MTT assay. (D) Colony formation assays of H1299-NC and H1299-CUL4A cells. (E) MTT assay and (F) colony formation assay of H460-NC and H460-shCUL4A cells. ${ }^{*} \mathrm{P}<0.05,{ }^{* *} \mathrm{P}<0.01$ vs. respective NC. CUL4A, cullin4A; OD, optical density; NC, negative control.

insight into the mechanism by which $C U L 4 A$ promotes bone metastasis of A549 cells, western blot analysis was used to examine expression of the EMT-activator ZEB1 and well-characterized EMT markers in lung adenocarcinoma cells (Fig. 5). The results showed that expression of the EMT-activator $Z E B 1$, and the mesenchymal marker vimentin, were markedly increased compared with the control, and expression of the epithelial marker E-cadherin was markedly decreased in lung adenocarcinoma cells overexpressing $C U L 4 A$, compared with the respective control cells. However, the expression levels of these proteins were reversed in the $C U L 4 A$-silenced lung adenocarcinoma cells. Therefore, these findings suggest that CUL4A may promote metastasis of lung adenocarcinoma to the bone via $Z E B 1$-mediated induction of $E M T$.

\section{Discussion}

In the present study it was shown that $C U L 4 A$ expression was associated with metastasis of lung adenocarcinoma. Upregulation of $C U L 4 A$ expression in lung adenocarcinoma cells increased proliferation, migration and invasion in vitro and increased metastasis to the bone in vivo. Conversely, silencing of $C U L 4 A$ resulted in the opposite effects in the H460 cells. Mechanistically, the transcriptional expression levels of $Z E B 1$ were associated with $C U L 4 A$ expression.

$C U L 4 A$ is a member of the evolutionarily conserved cullin family of proteins, which consists of seven-related cullins (Cull, Cul2, Cul3, Cul4A, Cul4B, Cul5, and Cul7) (12). $C U L 4 A$ forms part of the ubiquitin ligase E3 complex, and serves a crucial role in DNA replication, cell cycle regulation and genomic instability (13-17). Previous studies have demonstrated that $C U L 4 A$ acts as an oncogene in various types of tumors and promotes cancer development, including lung cancer, breast cancer, prostate cancer and other types of cancer $(7,18-20)$ and its upregulation is associated with less favorable outcomes (21), which further supports the results of the present study. Therefore, CUL4A may be a potential anticancer target due to the fact that several well-known tumor-suppressor genes, including $p 21, p 27$ and $p 53$, are ubiquitinated and degraded by the $C U L 4 A$-mediated E3 ubiquitin 
A
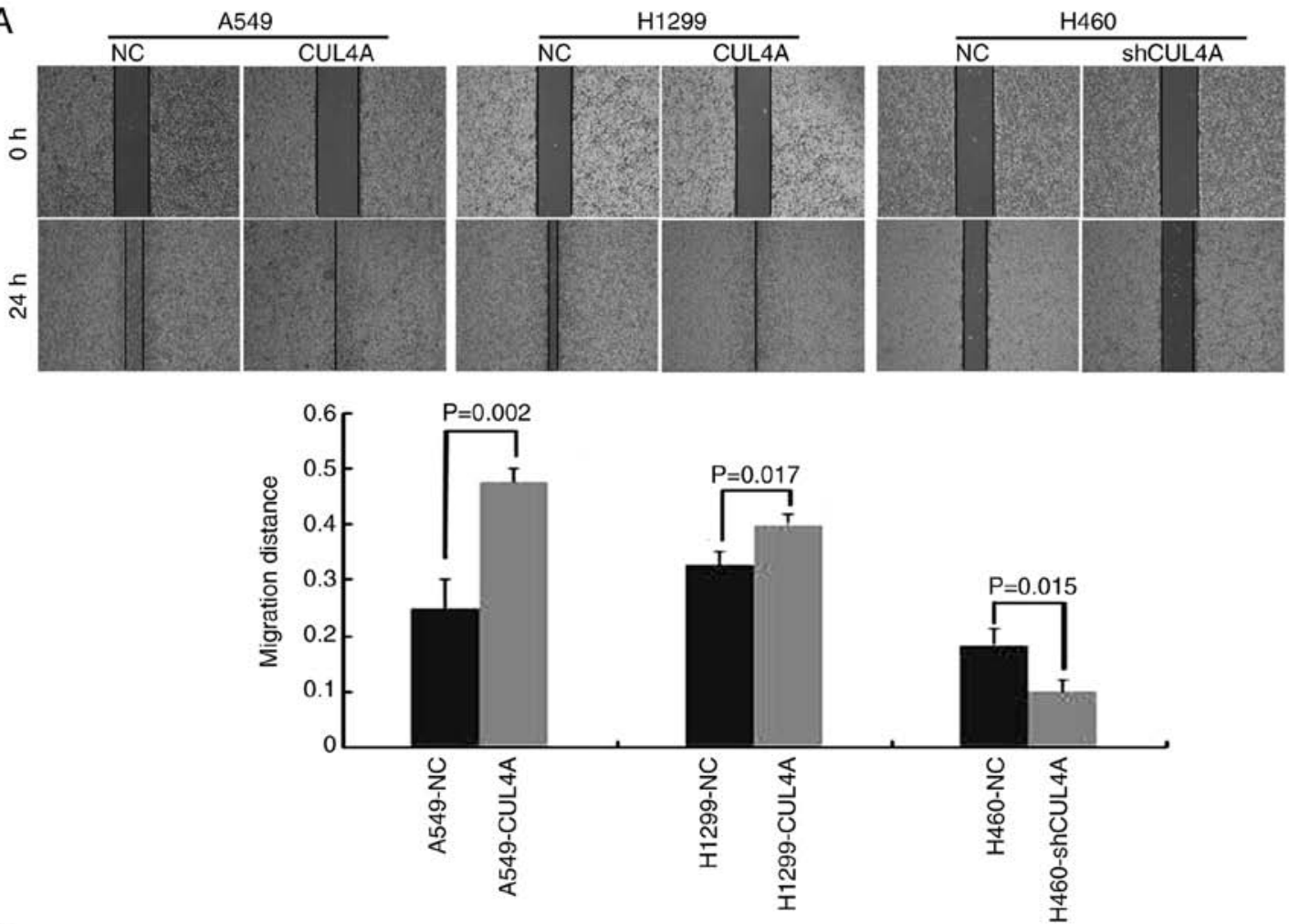

B
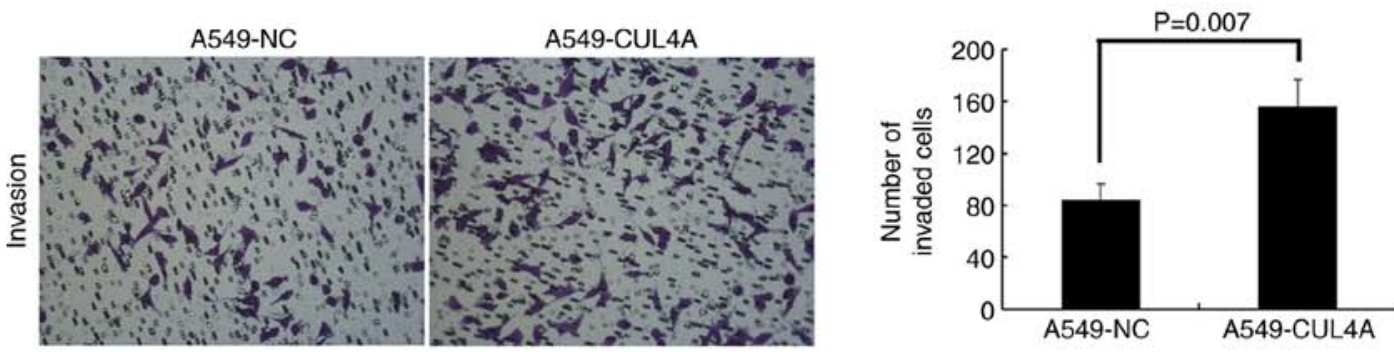

C

H1299-NC

H1299-CUL4A
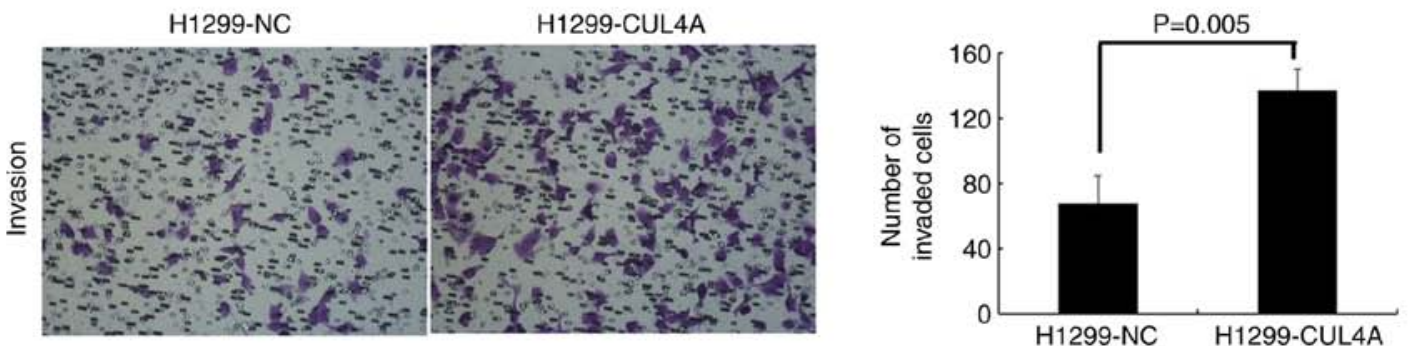

$\mathrm{D}$
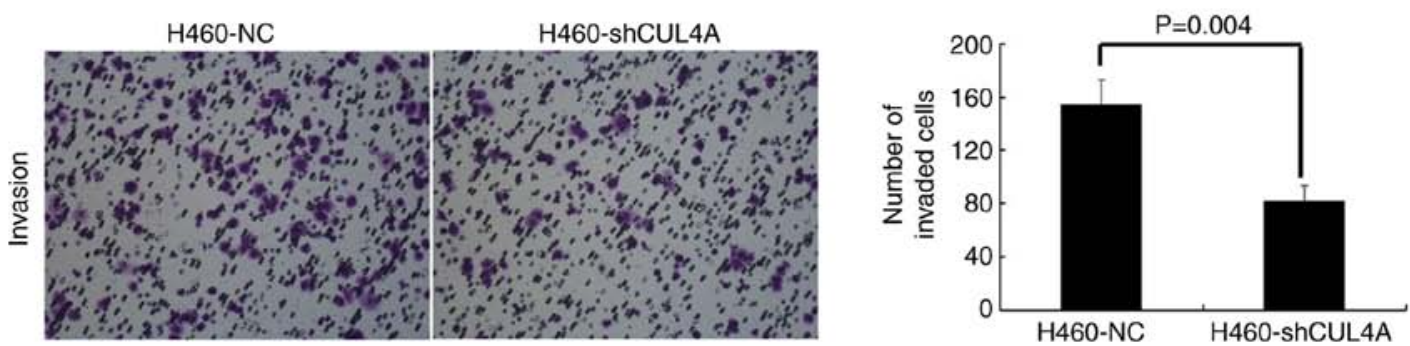

Figure 3. Effect of $C U L 4 A$ on metastasis of lung adenocarcinoma cells. (A) Representative images (x4 magnification) of a wound-healing assay. Representative images (x200) and statistical analysis of invasion of (B) A549-NC and A549-CUL4A, (C) H1299-NC and H1299-CUL4A, and (D) H460-NC and H460-shCUL4A cells in a Transwell invasion assay. Data are expressed as the mean \pm standard deviation. CUL4A, cullin4A; NC, negative control; sh, short hairpin.

proteasome system $(17,22,23)$. In a $C U L 4 A$-defcient mouse model of skin cancer, significantly increased resistance to UV-induced skin cancer was observed (24). In addition, a recent study reported that $C U L 4 A$ modulates invasion and metastasis of lung cancer through regulation of ANXA10 (25). Similarly, in the present study, it was demonstrated that $C U L 4 A$ overexpression served a significant role in promoting development of lung adenocarcinoma. The present study highlights 

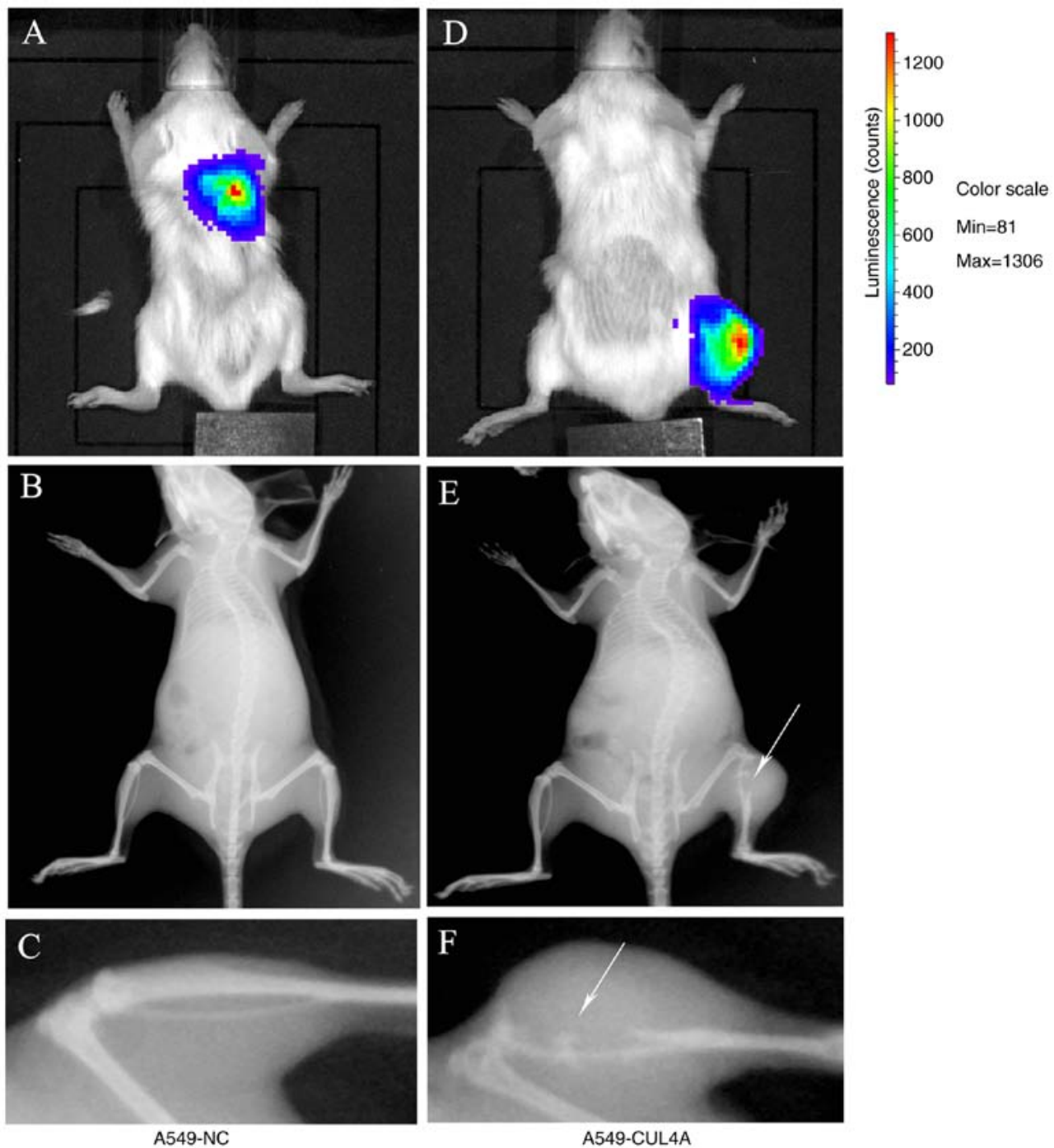

Figure 4. D-luciferin biofluorescence and representative X-ray images of bone metastasis in the mouse models. Control mice injected with A549-NC cells. Lung tumor development was assessed in vivo using (A) luciferase imaging and (B and C) normal bone tissues were detected using X-ray imaging. Experimental mice were injected with A549-CUL4A cells and metastasis to the bone was assessed in vivo using (D) luciferase imaging and as the arrows indicate (E and F) osteolytic bone metastasis lesions in the tibia in the mouse were visualized using X-ray imaging. NC, negative control; CUL4A, cullin4A.

Table I. Incidence of bone metastases and the number of metastatic lesions formed in the NOD-SCID mice.

\begin{tabular}{lcc}
\hline Cell line & Incidence & No. of bone metastases \\
\hline A549-NC & $1 / 5$ & $0.2 \pm 0.45$ \\
A549-CUL4A & $4 / 5$ & $1.6 \pm 1.14^{\mathrm{a}}$ \\
\hline
\end{tabular}

${ }^{\mathrm{a}} \mathrm{P}<0.05$ vs. control group. CULAA, cullin $4 A$.

a novel function of $C U L 4 A$ in metastasis of lung adenocarcinoma to the bone through transcriptional upregulation of the EMT-activator $Z E B 1$.

EMT is considered as the initial event during the development of metastasis of cancer, and is crucial for dissemination of tumor cells from primary sites and to colonize at distant tissues $(26,27)$. $Z E B 1$, a transcriptional repressor, is an essential inducer of EMT, and physiologically is required for the regulation of skeletal morphogenesis. Kerstin et al (28) reported that ZEB1 stimulates

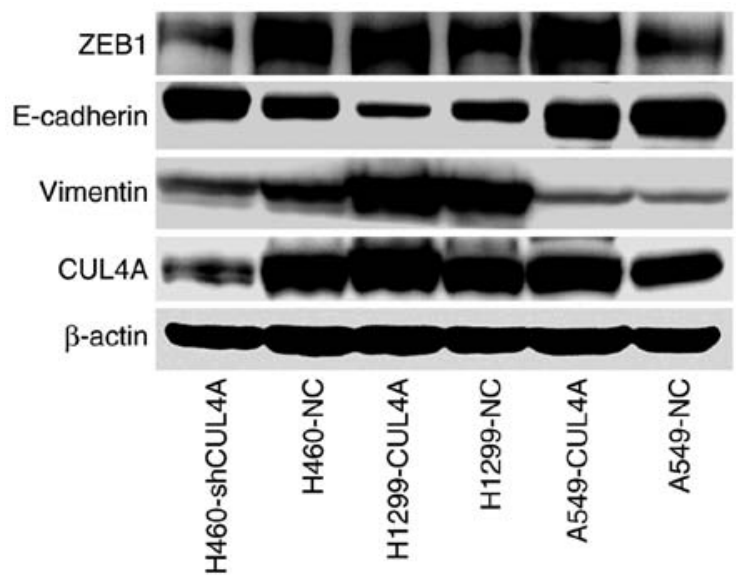

Figure 5. CUL4A mediates ZEB1-induced epithelial-mesenchymal transition. Expression of $Z E B 1$ and mesenchymal markers were upregulated and expression of epithelial markers were decreased in A549-CUL4A and H1299-CUL4A cells. Expression of mesenchymal proteins were decreased and expression of epithelial proteins were increased in the H460-shCUL4A cells compared with the H460-NC cells. CUL4A, cullin4A; ZEB1, zinc finger E-box binding homeobox 1; NC, negative control; sh, short hairpin. 
BMP-inhibitor mediated osteoclast differentiation and promotes metastasis of breast cancer to the bone. Studies have suggested that $Z E B 1$ may serve an import role in progression of lung adenocarcinoma $(29,30)$ and significantly increase metastasis of lung cancer to the bone (31), suggesting that ZEBI is a critical regulator of bone migration of lung cancer cells. Additionally, it has also been reported that $C U L 4 A$ transcriptionally activates ZEB1 through modulation of histone $\mathrm{H} 3 \mathrm{~K} 4 \mathrm{me} 3$, inducing EMT and promoting metastasis of breast cancer (32). CUL $4 A$ is associated with lung cancer cell proliferation and expression is associated with resistance to chemotherapy (20). However, the detailed mechanisms underlying CUL4A-mediated lung adenocarcinoma bone metastasis remain unknown. Therefore, in the present study, the means by which CUL $4 A$ induces EMT and promotes metastasis was examined, and it was demonstrated that $C U L 4 A$ is associated with $Z E B 1$ expression in lung adenocarcinoma cells, highlighting a potentially novel therapeutic target for prevention of bone metastasis in patients with lung cancer. The results of the present study showed that lung adenocarcinoma cells overexpressing CUL4A exhibited aggressive behaviors, including increased proliferation, migration and invasive capacities in vitro. Silencing of $C U L 4 A$ reversed these biological functions. In vivo, it was demonstrated that $C U L 4 A$ overexpression was significantly positively associated with increased bone metastatic lesions compared with the control group. These results are supported by Yang et al (31), Wang et al (32) and Kerstin et al (28), where it was demonstrated that ZEBI expression is positively associated with $C U L 4 A$ expression, and upregulation of $Z E B I$ expression promotes bone metastasis of lung and breast cancer. Therefore, $C U L 4 A$ may serve as a novel therapeutic target for prevention of metastasis of lung cancer to the bone.

Taken together, aberrant upregulation of $C U L 4 A$ expression upregulates the expression levels of $Z E B 1$, which in-turn increases expression of EMT-associated proteins and increases invasion and metastasis. This may underlie the mechanism by which $C U L 4 A$ increases metastasis of lung adenocarcinoma to the bone.

\section{Acknowledgements}

The authors are grateful for all the colleagues of the Oncology Research Center for their comments on earlier versions of this manuscript.

\section{Funding}

The present study was supported by the National Natural Science Foundation of China (nos. 81572251, 81572814 and 81902318).

\section{Availability of data and materials}

All data generated or analyzed during this study are included in this published article.

\section{Authors' contributions}

PPC and HLZ designed the study. PPC and WJC performed all the in vitro experiments and collected the data. HLP, WWS, PX,
LD and YXX conducted the animal experiments. HLP, WWS, PX, LD, YXX and LLL analyzed the data and performed the relative statistical analysis. LLL provided guidance during the study. PPC contributed to the writing of the manuscript. All authors have read and approved the final version of this manuscript and agree to be accountable for all aspects of the research in ensuring that the accuracy or integrity of any part of the work are appropriately investigated and resolved.

\section{Ethics approval and consent to participate}

All animal studies strictly abided by the Regulations on Animal Experimentation formulated by the Laboratory Animal Center of the Air Force Military Medical University (Xi'an, China) and this study was approved by the Animal Experimental Ethical Inspection Committee of this Center (no. 20181101).

\section{Patient consent for publication}

Not applicable.

\section{Competing interests}

The authors declare that they have no competing interests.

\section{References}

1. Lemjabbar-Alaoui H, Hassan OU, Yang YW and Buchanan P: Lung cancer: Biology and treatment options. Biochim Biophys Acta 1856: 189-210, 2015.

2. Reck M and Rabe KF: Precision diagnosis and treatment for advanced non-small-cell lung cancer. N Engl J Med 377: 849-861, 2017.

3. Siegel RL, Miller KD and Jemal A: Cancer statistics, 2019. CA Cancer J Clin 69: 7-34, 2019.

4. Allemani C, Matsuda T, Di Carlo V, Harewood R, Matz M, Nikšić M, Bonaventure A, Valkov M, Johnson CJ, Estève J, et al: Global surveillance of trends in cancer survival 2000-14 (CONCORD-3): Analysis of individual records for 37513025 patients diagnosed with one of 18 cancers from 322 populationbased registries in 71 countries. Lancet 391: 1023-1075, 2018.

5. Coleman RE: Clinical features of metastatic bone disease and risk of skeletal morbidity. Clin Cancer Res 12: 6243s-6249s, 2006.

6. Hannah J and Zhou P: Distinct and overlapping functions of the cullin E3 ligase scaffolding proteins CUL4A and CUL4B. Gene 573: 33-45, 2015.

7. Sharma $P$ and Nag A: CUL4A ubiquitin ligase: A promising drug target for cancer and other human diseases. Open Biol 4: $130217,2014$.

8. De Craene B and Berx G: Regulatory networks defining EMT during cancer initiation and progression. Nat Rev Cancer 13: 97-110, 2013

9. Thiery JP, Acloque H, Huang RY and Nieto MA: Epithelialmesenchymal transitions in development and disease. Cell 139: 871-890, 2009

10. Liu Y, Zhang N, Wang Y, Xu M, Liu N, Pang X, Cao J, Ma N, Pang H, Liu L and Zhang H: Zinc finger E-box binding homeobox 1 promotes invasion and bone metastasis of small cell lung cancer in vitro and in vivo. Cancer Sci 103: 1420-1428, 2012.

11. Livak KJ and Schmittgen TD: Analysis of relative gene expression data using real-time quantitative PCR and the 2(-Delta Delta $\mathrm{C}(\mathrm{T}))$ method. Methods 25: 402-408, 2001.

12. Zhong W, Feng H, Santiago FE and Kipreos ET: CUL-4 ubiquitin ligase maintains genome stability by restraining DNA-replication licensing. Nature 423: 885-889, 2003.

13. Lee $\mathrm{J}$ and Zhou P: Pathogenic role of the CRL4 ubiquitin ligase in human disease. Front Oncol 2: 21, 2012.

14. Sugasawa K: The CUL4 enigma: Culling DNA repair factors. Mol Cell 34: 403-404, 2009.

15. Han J, Zhang H, Zhang H, Wang Z, Zhou H and Zhang Z: A Cul4 E3 ubiquitin ligase regulates histone hand-off during nucleosome assembly. Cell 155: 817-829, 2013. 
16. Hu J and Xiong $\mathrm{Y}$ : An evolutionarily conserved function of proliferating cell nuclear antigen for Cdt1 degradation by the Cul4-Ddb1 ubiquitin ligase in response to DNA damage. J Biol Chem 281: 3753-3756, 2006.

17. Li B, Jia N, Kapur R and Chun KT: Cul4A targets p 27 for degradation and regulates proliferation, cell cycle exit, and differentiation during erythropoiesis. Blood 107: 4291-4299, 2006.

18. Xu Y, Wang Y, Ma G, Wang Q and Wei G: CUL4A is overexpressed in human pituitary adenomas and regulates pituitary tumor cell proliferation. J Neurooncol 116: 625-632, 2014.

19. Melchor L, Saucedo-Cuevas LP, Muñoz-Repeto I, RodríguezPinilla SM, Honrado E, Campoverde A, Palacios J, Nathanson KL, García MJ and Benítez J: Comprehensive characterization of the DNA amplification at 13q34 in human breast cancer reveals TFDP1 and CUL4A as likely candidate target genes. Breast Cancer Res 11: R86, 2009.

20. Wang Y, Zhang P, Liu Z, Wang Q, Wen M, Wang Y, Yuan H, Mao JH and Wei G: CUL4A overexpression enhances lung tumor growth and sensitizes lung cancer cells to erlotinib via transcriptional regulation of EGFR. Mol Cancer 13: 252, 2014

21. Birner P, Schoppmann A, Schindl M, Dinhof C, Jesch B Berghoff AS and Schoppmann SF: Human homologue for Caenorhabditis elegans CUL-4 protein overexpression is associated with malignant potential of epithelial ovarian tumours and poor outcome in carcinoma. J Clin Pathol 65: 507-511, 2012.

22. Nishitani H, Shiomi Y, Iida H, Michishita M, Takami T and Tsurimoto T: CDK inhibitor $\mathrm{p} 21$ is degraded by a proliferating cell nuclear antigen-coupled Cul4-DDB1Cdt2 pathway during $\mathrm{S}$ phase and after UV irradiation. J Biol Chem 283: 29045-29052, 2008.

23. Nag A, Bagchi S and Raychaudhuri P: Cul4A physically associates with MDM2 and participates in the proteolysis of $\mathrm{p} 53$. Cancer Res 64: 8152-8155, 2004.

24. Liu L, Lee S, Zhang J, Peters SB, Hannah J, Zhang Y, Yin Y, Koff A Ma L and Zhou P: CUL4A abrogation augments DNA damage response and protection against skin carcinogenesis. Mol Cell 34 451-460, 2009.
25. Hung MS, Chen YC, Lin P, Li YC, Hsu CC, Lung JH, You L, $\mathrm{Xu} \mathrm{Z}$, Mao JH, Jablons DM and Yang CT: Cul4A modulates invasion and metastasis of lung cancer through regulation of ANXA10. Cancers (Basel) 11: pii: E618, 2019.

26. Prudkin L, Liu DD, Ozburn NC,Sun M,BehrensC, Tang X, Brown KC, Bekele BN, Moran C and Wistuba II: Epithelial-to-mesenchymal transition in the development and progression of adenocarcinoma and squamous cell carcinoma of the lung. Mod Pathol 22: 668-678, 2009.

27. Cowin P, Rowlands TM and Hatsell SJ: Cadherins and catenins in breast cancer. Curr Opin Cell Biol 17: 499-508, 2005.

28. Mock K, Preca BT, Brummer T, Brabletz S, Stemmler MP and Brabletz T: The EMT-activator ZEB1 induces bone metastasis associated genes including BMP-inhibitors. Oncotarget 6 : 14399-14412, 2015

29. Takeyama Y, Sato M, Horio M, Hase T, Yoshida K, Yokoyama T, Nakashima H, Hashimoto N, Sekido Y, Gazdar AF, et al: Knockdown of ZEB1, a master epithelial-to-mesenchymal transition (EMT) gene, suppresses anchorage-independent cell growth of lung cancer cells. Cancer Lett 296: 216-224, 2010

30. Gemmill RM, Roche J, Potiron VA, Nasarre P, Mitas M Coldren CD, Helfrich BA, Garrett-Mayer E, Bunn PA and Drabkin HA: ZEB1-responsive genes in non-small cell lung cancer. Cancer Lett 300: 66-78, 2011.

31. Yang X, Li L, Huang Q, Xu W, Cai X, Zhang J, Yan W, Song D, Liu T, Zhou W, et al: Wnt signaling through Snaill and Zeb1 regulates bone metastasis in lung cancer. Am J Cancer Res 5: 748-755, 2015.

32. Wang Y, Wen M, Kwon Y, Xu Y, Liu Y, Zhang P, He X, Wang Q, Huang Y, Jen KY, et al: CUL4A induces epithelial-mesenchymal transition and promotes cancer metastasis by regulating ZEB1 expression. Cancer Res 74: 520-531, 2014. 